DOI: https://doi.org/10.47405/mjssh.v5i12.555

\begin{tabular}{|c|c|}
\hline Ins & Malaysian Journal of Social Sciences and Humanities (MJSSH) \\
\hline Malaysian Journal of & Volume 5, Issue 12, December 2020 \\
\hline (MJ-SSH) & e-ISSN : 2504-8562 \\
\hline & $\begin{array}{l}\text { Journal home page: } \\
\text { www.msocialsciences.com }\end{array}$ \\
\hline
\end{tabular}

\title{
Exploring the Knowledge on Herbal Medicine (HM) usage in Disease Management among People with Type 2 Diabetes Mellitus (T2DM) in Negeri Sembilan, Malaysia
}

\author{
Rosnani Kassim¹, Norbaidurah Ithnain'1, Albeny Joslyn Panting1, Nadia Amirudin1, \\ Manimaran Krishnan ${ }^{1}$ \\ ${ }^{1}$ Institute for Health Behavioural Research, Ministry of Health Malaysia
}

Correspondence: Rosnani Kassim(rosnani_k@moh.gov.my)

\begin{abstract}
It is estimated that diabetes affects approximately 415 million people worldwide and this is expected to increase to 642 million by 2040 . There are several methods used in managing diabetes which include nutritional control, taking medications, insulin injection and/or consuming herbal medicine (HM). This study explored the knowledge of herbal medicine usage among people with Type 2 Diabetes Mellitus (T2DM) in regard to diabetes management. A qualitative study was conducted among 28 diabetic patients from government clinics in Negeri Sembilan. They were interviewed face-to-face and all information about their herbal medicine consumption were analysed thematically. Findings showed that the main source of information on herbal usage were from family members, friends and/or neighbours. This study discovered that knowledge on herbal medicine among T2DM patients was greatly influenced from such sources and it is also hereditary. In addressing herbal medicine safety issues, some patients were unaware of the proper channel while others chose to accept any arising consequences without informing the healthcare authorities. This study also revealed that most of the informants were aware on the types and ways of consuming herbal medicine. Besides, patients understood that diabetes can only be controlled and cannot be cured. However, knowledge on complementary intake with modern medicine should be enlightened. Therefore, it is suggested that the healthcare provider need to update their knowledge on herbal medicines and be able to give informative insights to their patients.
\end{abstract}

Keywords: knowledge, herbal medicine, type 2 diabetes patients

\section{Introduction}

Diabetes is a metabolic disorder resulting either from defects in insulin secretion by the pancreas or body's ineffective in insulin utilisation. Insulin is a hormone that modulate blood sugar balance in the body. Disturbance in insulin production will cause hyperglycemia or raised blood sugar level and over time leads to serious damage to many of the body's systems, especially the nerves and blood vessels (Alberti \& Zimmet, 1998). Diabetes is classified into Type 1 and Type 2. Type 2 Diabetes Mellitus (T2DM) or previously referred as non-insulin dependent diabetes is a condition where the body doesn't produce enough insulin or ineffective use of insulin caused by many different factors (American Diabetes Association [ADA], 2004).

Diabetes can be treated and its consequences can be avoided or delayed through lifestyles modification; diet, physical activity, medication as well as regular screening and treatment for complications 
(International Diabetes Federation [IDF], 2019). Additionally, several studies mentioned complementary and alternative medicines (CAM) usage in managing diabetes (Naghibi Harat, Jalali, Zarafshan, Ebadiani, \& Karbakhsh, 2008; Thomson, Jones, Evans, \& Leslie, 2012). Perception on the side effects and ineffectiveness of conventional medicines and the belief that traditional herbal medicine (HM) is much better than modern medicine lead to the CAM usage (Naghibi Harat et al., 2008).

Traditional and Complementary Medicine (T\&CM) is the combination of the words traditional medicine and complementary medicine, found in almost every nation worldwide and the demand for its services is growing. Traditional medicine has been defined as "a sum total of the knowledge, skills and practices based on the theories, beliefs and experiences indigenous to various cultures, whether interpretable or not, used in the maintenance of health as well as prevention, diagnosis, betterment or care of physical and mental illnesses/wellness" (World Health Organisation [WHO], 2019, p.9). Complementary medicine or also known as alternative medicine refers to a broad set of health care practices that are not part of the country's own tradition or conventional medicine and are not fully integrated into the dominant healthcare system (Maimunah, Ramli, Shamsaini, \& Jaafar, 2011).

Based on the National Health and Morbidity Survey (NHMS) (2015), T\&CM practices were mainly used to maintain wellness and among those were Malay massage (41.92\%), Chinese herbs (13.69\%), Malay herbs $(10.07 \%)$, Malay cupping $(6.45 \%)$ and Tuina $(4.49 \%)$. These practices were often used for minor illnesses such as myalgia, joint and muscle ache, back pain and cough. The users obtained services of T\&CM at various facilities depending on the practices. A study by Siti et al. (2009) showed a prevalence of $69.4 \%$ of the Malaysian population had ever used T\&CM in their lifetime and 55.6\% had used T\&CM within the last 12-month period of the survey.

Globally, approximately 463 million adults (20-79 years old) were living with diabetes and the amount of T2DM diabetic patients is increasing. In 2019, 374 million people were at an increasing risk of developing T2DM. A survey by the Ministry of Health, Malaysia demonstrated a prevalence (known or undiagnosed T2DM) of $17.5 \%$ adults aged 18 years and above. The prevalence showed an increasing trend with age, from 5.5\% in the 18-19 years old age group, reaching a peak of 39.1\% among the 70-74 years old age group. Among the known diabetic patients, $0.5 \%$ preferred T\&CM as their treatment (NHMS, 2015).

A study by Kalantzi, Kostagiolas, Kechagias, Niakas, and Makrilakis (2015), mentioned that information and knowledge from the patients' perspective has an important role to diabetes management and as a result, diabetes self-management education (DSME) and continuous support were significant contributors to metabolic and psychological outcomes. This is in line with Zhang et al. (2019), regarding the needs for patient to have the ability to find relevant quality health information in order to participate in medical decision-making. However, inadequate knowledge among doctors on CAM and the absence of regulation hindered them from giving informed advice to their patients on herbal usage (Botting \& Cook, 2000; Ameade, Amalba, Helegbe, \& Mohammed, 2015).

\section{Methods}

\section{Study Design}

This qualitative study was conducted to explore the knowledge on Herbal Medicine (HM) among Type 2 Diabetes Mellitus (T2DM) patients. Two clinics from the rural areas (Klinik Kesihatan Kuala Pilah and Klinik Kesihatan Bahau) and two clinics from the urban areas (Klinik Kesihatan Seremban and Klinik Kesihatan Port Dickson) of Negeri Sembilan, Malaysia were selected for this study. These four clinics were selected based on the Report on Diabetic Patient's Information (2016) that reported the highest number of diabetes registration. Informants were interviewed from August 2017 till March 2018 through purposive sampling. A guided semi-structured questionnaire was prepared for face-to-face interview consisting questions on source of information and knowledge on the usage of herbal medicine. All interview sessions were digitally recorded with permission from the informant prior to the interview session and notes were taken. 


\section{Data Analysis}

The contents of the interview sessions were transcribed verbatim and transcriptions were reviewed for accuracy. The analysis was conducted during the fieldwork, when initial impressions of both potential themes and the direction of the research were noted. This study used line-by-line coding to code every single line. This study also used open coding as there are no pre-set codes. The codes were developed and modified during the coding process followed with peer checking. Final analysis was conducted after the thematic analysis.

\section{Ethical Consideration}

Permission to conduct this study was obtained from the National Medical Research Registry (NMRR) and the heads of departments of the participating clinics. Ethical approval was given by the Medical Research Ethics Committee, Ministry of Health (NMRR ID: 17-953-35849). All participants were informed and was given written consent forms before the commencement of the interviews. All the participants also granted approval on the audio recording prior to the interviews.

\section{Results}

\section{Characteristics of the Informants}

The informants consisted of 15 males and 13 females with a total number of 28 participants. The multiracial groups consisted of Malay, Chinese and Indians. Inclusion criteria for the informants were patients diagnosed with diabetes and having treatment with health clinics for a minimum of three years and more, as well as have taken or even tried HM in managing their diseases.

\section{Themes}

The following themes were extracted from the thematic analysis: (a) source of knowledge about herbal medicine, (b) knowledge on channel to address regarding herbal medicine safety issues, (c) knowledge about types/forms and consumption process of herbs taken, and (d) knowledge regarding diabetes can be cured.

\section{a) Sources of knowledge aboutherbalmedicine}

This theme addressed the source of knowledge among the informants on HM. In general, fenugreek is widely used by Malaysians as one of the ingredients in daily cooking. Most of the herbs mentioned were familiar among family, friends and neighbours.

\footnotetext{
"My mother used to consume and she said it is good for health and because it is more on the traditional thing you know (taking fenugreek)..." (Informant 12, Female, Indian, 63)

"From friends, they introduce cat's whiskers..." (Informant 18, Female, Chinese, 57)

"There was once, my neighbour was diabetic... he had and suggested to us... we recommended to him, like that..." (Informant 26, Female, Malay, 52)
}

Apart from that, they knew about HM from prime media such as television, radio and newspaper.

"It's Indian herbal specialist Doctor lah. I watched on TV and follow his program on the Astro channel and tried it." (Informant 28, male, Indian, 64) 
"This what we saw in the newspaper. Read that newspaper (stevia)." (Informant 3, Female, Indian, 66)

A few of the informants gained knowledge on HM through the internet and social media, such as YouTube, Facebook and other forms of social media. Some of them received information through Facebook on the benefits of green apple or bitter gourd in managing T2DM. Transformation in technology makes information easy to access for everyone at anytime and anywhere. Social media become more popular for all ages in exploring information and developing desired skills. It is also one of the fastest spreading mediums of information and testimonial sharing which can reach and influence many people.

"Youtube. And then this one.. a lot of Bengali people also taking you know." (Informant 3, Female, Indian, 66)

"People say green apples for blood pressure are good. Bitter gourd, on Facebook a lot of information on bitter gourd..." (Informant 12, Male, Malay, 64)

\title{
b) Knowledge on channel to address regarding herbal medicine safety issue
}

The informants were asked about knowledge regarding how and where to report a complaint if they experience any side effects on herbal consumption. Some informants had no knowledge on whom to report regarding the side effects from the usage of HM. However, some stated that they will not report to anybody because they felt responsible for the choices made in using HM to manage their diseases. Many of them informed that they would stop consuming once any side effects occurred from the HM usage.

"I don't know who to report, where to report." (Informant 11, Male, Malay, 52)

"No, I'm not. I risk that I have my own fault. I will not complain to anyone, it is my will, it is my responsibility." (Informant 1, Male, Indian, 62)

"No..no. This is not people force you to take maa. So you just want to try try only." (Informant 18, Female, Chinese,57)

“No ... After that, I will not consume anymore." (Informant 3, Female, Indian, 66)

\section{c) Knowledge aboutforms, content and safety of herbs taken}

Most of the informants were aware on the types/forms of HM they consumed and methods of consumption.

\author{
"haa, it's like flakes... I like to take 3, 2 pieces, 4 pieces. It's dry." (Informant 11, \\ Male, Malay, 52)
}

"That day went to Alpro Pharmacy and bought bitter gourd capsule, and its good." (Informant 15, Female, Malay, 50)

"Read, read labels in writing. There is a guide on how much he should take, how many doses, how many times can he mix with water, so read on." (Informant 3, Female, Indian, 66)

\section{d) Knowledge regarding diabetes can be cured}

Besides the knowledge of $\mathrm{HM}$, this study also explores the knowledge regarding the cure for diabetes among informants. Majority of the informants had a good understanding of their diseases and realised that diabetes mellitus cannot be cured but can be managed with a healthy lifestyle including controlled 
eating habits, physical activities and disciplined in medicine intake. However, some believed that their diseases can be cured.

"That he said diabetes is manageable, no cure for diabetes, just controlled." (Informant 10, Male, Malay, 47)

"No one ever talks about healing. Until death take the medicine." (Informant 28, Male, Chinese, 75)

"Can be healed, it's GOD's will I think I am going to be cure .. haaa God's will, I am confident." (Informant 11, Male, Malay, 52)

\section{Discussion}

The present study was designed to explore the knowledge of herbal medicine and the usage in disease management among patients with T2DM. Qualitative analysis from T2DM patients' knowledge on HM revealed four major themes; (a) source of knowledge about HM, (b) knowledge on channel to address regarding herbal medicine safety issues, (c) knowledge about types/forms and consumption process of herbs taken, and (d) knowledge regarding diabetes can be cured.

In general, family and friends were the primary sources of knowledge regarding HM. In terms of HM consumption, Malaysian's culture in society and verbal information still maneuvers a major role in disseminating knowledge about HM among patients with T2DM. It showed that family and peers had a very coercive influence factor to the informants for this study to use HM as part of their disease management. Our multiracial culture also played a part in sharing either positive or negative information of HM usage and to some extent acted like an expert. Some of the practices has been used, in particular race and were shared among them. Ching, Zakaria, Paimin, \& Jalalian (2013), found that most respondents learned about T\&CM mainly from friends and family members followed by media and health personal. Similarly, L.L. Low, Tong, and W.Y. Low (2016), cited the same source of information which was from social groups such as family members, friends, peers and health care staff. The study found that patients with T2DM actively interacted among their social group in the process of selecting treatment. The influential impacted from the social group was reckoned from the strength of the relationship, including the level of trust, and the support they received.

Additionally, prime media; television, radio, newspaper, books and magazines are still being used by the public to access information on HM. As majority of the informants were 50 to 75 years old, the possibility for this group to seek and obtain better knowledge on HM through prime media were most probably due to their accustomed with the traditional mode of getting information. This was supported by Hasan et al. (2011), where the internet was the most commonly used source of information on T\&CM among the younger participants (average of 21 years old). Besides the internet, people with diabetes also gained knowledge about diabetes through books and brochures (Low et al., 2016).

This study also found that some informants had no knowledge on the channel to address any side effect issues from using HM. Some might secretly consume HM to avoid from getting blamed and rather bear any consequences from HM usage, than complaining to the respective authority. As most of the participants are at the senior ages, psychological or other approaches may be considered to assist and ease them in addressing the complaints correctly. The majority of informants claimed that the herbs they consumed were safe because it is from natural sources. Without knowing the effectiveness and safety of the products taken, they will not complain as it is self-chosen. According to Babar, Syed, Naing, and Hamzah (2012), insufficient knowledge of T\&CM toxicity due to scarce of proper reference resources might cause the public to become unaware of T\&CM side effects. Similarly, a study by Silvanathan et al. (2015), found that approximately half of the respondents were unaware of the possible side effects and herb-drug interactions of T\&CM so they might perceive that T\&CM has lower rate of side effects than conventional Western treatments, hence many consumers might change to T\&CM products. 
In terms of knowledge about forms, content and safety of herbs taken, some informants were aware of the HM that they consumed. Various HM information from the elderly delivered through generations without knowledge of the unknown side effects, therefore most were unaware of the contents of the product consumed for years. Given that, it was their choice of treatment, they would accept any consequences. According to Ching et al. (2013), the higher consumption was predictable since most people with diabetes presumed that herbs are safer, affordable and accessible. In addition, the high consumption of HM reported were deeply rooted and influenced by its multicultural and religious nature. A study discovered that the most common advantages of CAM usage were no side effects, more convenient and showed a complete cure, whereas the disadvantages include effective only for limited diseases, expensive, need food restrictions and gave symptomatic relief only (Jaiswal et al., 2015).

According to T\&CM Division (2017), a limited source of information on T\&CM were something that should be interpreted seriously by the authorities. Any campaign or promotion by the authority might be useful for the public to understand and aware about T\&CM. There is demand for T\&CM as people tend to look for alternative treatment that can be cured. T\&CM is common because it has been used for ages before modern medicine came into the picture. T\&CM practices has been culturally used and became part of home remedies in curing signs and symptoms of common diseases. Furthermore, patients from this study seem to look for better unconventional medicine in managing T2DM, particularly HM.

Another key finding obtained from this study was on knowledge regarding diabetes can be cured. Majority of our informants had a great understanding of their diseases and acknowledge that diabetes mellitus cannot be cured, somehow can be controlled with a healthy lifestyle.

\section{Conclusion}

This study explored the knowledge of HM usage in disease management among people with T2DM. Family and friends were the most influential source on the decision of the T2DM patients on the usage of HM. Family members and friends are those who have close relationships and frequently communicate with patients; therefore, it is necessary to include relatives and friends when discussing the problem and treatment for diabetes with the patients.

A better understanding on the usage of HM will help the medical professionals be more vigilant and patient-centered, particularly during counselling sessions regarding the proper use of herbal remedies in the stream of modern medicine. As a result, health care providers should acquaint themselves with the knowledge on common HM for diabetes, their mechanism of action, drug-to-drug interactions and their likely adverse effects.

Awareness on safety and quality of HM products either in the form of plants or end products, should be taken into consideration for further research and development. This is important, because with the precise resource of information and knowledge, it will help our society including the patients' caretaker to decide what kind of HM and in what amount should be taken by the patients in managing their T2DM for a better quality of life.

In conclusion, public preference for HM is higher but there was a lack of awareness on the side effects. Since the generality of HM is high, health-care professionals may need to improve their knowledge on $\mathrm{HM}$ and their ability to manage the possibility of any adverse events. Public education on the safety of $\mathrm{HM}$ is also needed to be taken into consideration.

\section{Acknowledgement}

We would like to thank the Director-General of Health and Deputy Director-General of Health (Research and Technical Support), Ministry of Health, Malaysia for the permission to publish this paper. 


\section{Competing Interest Statement}

The authors declared that there are no competing or potential conflicts of interest.

\section{References}

Alberti, K. G. M. M., \& Zimmet, P. Z. (1998). Definition, diagnosis and classification of diabetes mellitus and its complications. Part 1: diagnosis and classification of diabetes mellitus. Provisional report of a WHO consultation. Diabetic medicine, 15(7), 539-553.

Ameade, E. P., Amalba, A., Helegbe, G. K., \& Mohammed, B. S. (2015). Herbal medicine: a survey on the knowledge and attitude of medical students in Tamale, Ghana. Peak Journal of Medicinal Plant Research, 3(1).

American Diabetes Association. (2004). Diagnosis and Classification of Diabetes. Diabetes Care, 27, (Supplement 1).

Babar, M. G., Syed, S. H., Naing, C. M., \& Hamzah, N. H. B. (2012). Perceptions and self-use of Complementary and Alternative Medicine (CAM) among Malaysian dental students. European Journal of Integrative Medicine, 4(1), e63-e69.

Botting, D. A., \& Cook, R. (2000). Complementary medicine: knowledge, use and attitudes of doctors. Complementary Therapies in Nursing and Midwifery, 6(1), 41-47.

Ching, S. M., Zakaria, Z. A., Paimin, F., \& Jalalian, M. (2013). Complementary alternative medicine used among patients with type 2 diabetes mellitus in the primary care setting: a cross-sectional study in Malaysia. BMC Complementary and Alternative Medicine, 13(1), 1-7.

Hasan, S. S., Yong, C. S., Babar, M. G., Naing, C. M., Hameed, A., Baig, M. R., ... \& Kairuz, T. (2011). Understanding, perceptions and self-use of complementary and alternative medicine (CAM) among Malaysian's pharmacy students. BMC complementary and alternative medicine, 11(1), 95.

Institute for Public Health (IPH). (2015). National Health and Morbidity Survey 2015 (NHMS 2015). Vol. II: Non-Communicable Diseases, Risk Factors \& Other Health Problems.

International Diabetes Federation (IDF) (2020, March 20). Available at: https://www.idf.org/aboutdiabetes/type-2-diabetes.html (Accessed on 23rd June 2020).

Jaiswal, K., Bajait, C., Pimpalkhute, S., Sontakke, S., Dakhale, G., \& Magdum, A. (2015). Knowledge, attitude and practice of complementary and alternative medicine: A patient's perspective. International Journal of Medicine and Public Health, 5(1).

Kalantzi, S., Kostagiolas, P., Kechagias, G., Niakas, D., \& Makrilakis, K. (2015). Information seeking behaviour of patients with diabetes mellitus: a cross-sectional study in an outpatient clinic of a university-affiliated hospital in Athens, Greece. BMC Research Notes, 8(1), 48. doi:10.1186/s13104-015-1005-3

Low, L. L., Tong, S. F., \& Low, W. Y. (2016). Selection of treatment strategies among patients with type 2 diabetes mellitus in Malaysia: a grounded theory approach. PLoS One, 11(1), e0147127.

Maimunah, A. H., Ramli, A. G., Shamsaini, S., \& Jaafar, L. (2011). A handbook of traditional and complementary medicine programme in Malaysia: Traditional and Complementary Medicine. Kuala Lumpur: Ministry of Health Malaysia.

Naghibi Harat, Z., Jalali, N., Zarafshan, M., Ebadiani, M., \& Karbakhsh, M. (2008). A glance on Iranian traditional medicine and determining the medical student's perspective. Iranian Journal of Medical Ethics and History of Medicine, 1(3), 45-54.

Silvanathan, S., \& Low, B. S. (2015). Current public awareness on the safety of traditional and complementary medicines (T\&CM) in Malaysia. European Journal of Integrative Medicine, 7(2), 184-189.

Siti, Z. M., Tahir, A., Farah, A. I., Fazlin, S. A., Sondi, S., Azman, A. H., ... \& Zakiah, I. (2009). Use of traditional and complementary medicine in Malaysia: a baseline study. Complementary Therapies in Medicine, 17(5-6), 292-299.

Thomson, P., Jones, J., Evans, J. M., \& Leslie, S. L. (2012). Factors influencing the use of complementary and alternative medicine and whether patients inform their primary care physician. Complementary Therapies in Medicine, 20(1-2), 45-53. 
DOI: https://doi.org/10.47405/mjssh.v5i12.555

Traditional and Complementary Medicine Division (2017). Traditional and Complementary Medicine Blueprint 2018-2027. Ministry of Health Malaysia.

World Health Organisation. (2019). Global report on Traditional and Complementary Medicine.

Zhang, X., Foo, S., Majid, S., Chang, Y.-K., Dumaual, H. T. J., \& Suri, V. R. (2020). Self-care and health- information seeking behaviours in diabetic patients in Singapore. Journal of Health Communication, 35(8), 994-1003, DOI: 10.1080/10410236.2019.1606134 